\title{
The Calming Effect of a Maternal Breast Milk Odor on the Human Newborn Infant
}

Shota Nishitani ${ }^{1}$, Tsunetake Miyamura ${ }^{2}$, Masato Tagawa $^{3}$, Muneichiro Sumi $^{3}$, Ryuta Takase ${ }^{1}$, Hirokazu Doi ${ }^{1}$, Hiroyuki Moriuchi ${ }^{3}$ and Kazuyuki Shinohara ${ }^{1}$

${ }^{1}$ Department of Neurobiology and Behavior, Unit of Basic Medical Sciences, Course of

Medical and Dental Sciences, Nagasaki University, Graduate School of Biomedical Sciences, 1-12-4 Sakamoto, Nagasaki 852-8523, Japan

${ }^{2}$ Miyamura Obstetrics and Gynecology Clinic, 22-10, Hashiguchi-machi, Nagasaki 852-8114, Japan

${ }^{3}$ Department of Pediatrics, Nagasaki University Hospital, 1-7-1 Sakamoto, Nagasaki 852-8501, Japan

Correspondence to be sent to: Kazuyuki Shinohara, Department of Neurobiology and Behavior, Unit of Basic Medical Sciences, Course of Medical and Dental Sciences, Nagasaki University, Graduate School of Biomedical Sciences, 1-12-4 Sakamoto, Nagasaki, Japan. e-mail: kazuyuki@nagasaki-u.ac.jp

Total number of pages: 27

Total number of figures: 2

Total number of tables: 2 


\section{Abstract}

We examined the effects of the odors from mother's milk, other mother's milk and formula milk on pain responses in newborns undergoing routine heelsticks. Forty-eight healthy infants were assigned to four groups, an own mother's breast milk odor group (Own MM), another mother's breast milk odor group (Other MM), a formula milk odor group (Formula M) and a control group. To assess infant distress in response to the heelsticks, their crying, grimacing and motor activities were recorded during the experiment as behavioral indices of the pain response. After the heelstick, the behavioral indices of the Own MM group were lower than those of other groups. By contrast, the Other MM and Formula M groups showed no significant changes compared with the Control group. We also measured salivary cortisol concentration as a biochemical index in Control and Own MM infants before and after heelstick. After the heelstick, the level of salivary cortisol was significantly increased in Control infants, but not in Own MM infants. These results suggest that pain is relieved in human newborns when they are exposed to odors from their mother's milk.

KEY WORDS: newborns, olfaction, milk odors, stress, pain relief, behavior 


\section{Introduction}

The olfactory system is more mature at birth than the other senses. Mamm alian neonates must reach nipples by themselves to survive in the new environment outside the uterus (Winberg and Porter, 1998). Human newborns within the first days of life can detect and recognize the odor of their mother's nipples to get breast milk without any experience of feeding (Russell, 1976; Schaal et al., 1980; Varendi et al., 1994). Breast-feeding infants at postnatal day 4 to 15 have been shown to prefer the odor of their mother's breast to the breast odor of an unfamiliar lactating mother (Macfarlane, 1975; Makin and Porter, 1989). Similar discrimination between maternal and non-maternal odors from the neck, axillary and whole body is observed in neonates at 1-10 days of age (Cernoch and Porter, 1985; Sullivan and Toubas, 1998). Taken together, these results demonstrate that infants are precociously attracted to body odors associated with their mother and their mother's breast milk.

Studies on the short-term effects of pain have shown that pain affects various physiological and behavioral parameters. Regarding physiological parameters, there are increases in cardiac rhythm and plasma cortisol levels, and decreases in oxygen level.

Behavioral activities, such as crying, grimacing and motor activity, are increased (Craig et al., 1993; Johnston et al., 1996; Johnston et al., 1995). Using these parameters, soothing and analgesic methods have been developed such as nonnutritive suckling, sucrose, skin-to-skin contact, and breast feeding (Gray et al., 2000; Johnston et al., 2002). 
Recently, the soothing effects of maternal odors on newborns were examined in several studies (Mellier et al., 1997; Rattaz et al., 2005; Sullivan and Toubas, 1998). The pain expression of infants more quickly returned to basal levels when maternal milk odors were presented during a painful procedure. Rattaz and colleagues (2005) reported that the pain responses of infants, such as head movements, were decreased when they were presented with odors from their mother's milk. However, the effects of odors from another mother's milk or formula milk were not examined in their report. In the present study, we examined the effects of odors from mother's milk, other mother's milk and formula milk on infants' behavioral pain responses, such as crying, grimacing and motor activity, to compare the effects of these milk odors. We also measured salivary cortisol as a biochemical index of the pain response before and after heelstick, when infants were presented with the odor of their mother's milk.

\section{Materials and Methods}

\subsection{Subjects}

Experiment 1

Forty-eight rooming-in newborns on postnatal day 5 were recruited from the Miyamura Obstetrics and Gynecology Clinic and the Department of Pediatrics of Nagasaki University

Hospital. The selection criteria included term delivery (that is, gestational age 38-41 weeks), 
absence of gestational or perinatal complications (Apgar score $>8$ at $1 \mathrm{~min}$, and Apgar score $=10$ at 5 and $10 \mathrm{~min}$ after expulsion) and absence of neonatal pathologies or defects. The subjects consisted of 28 boys and 20 girls. The general characteristics of the newborn and mother are given in Table 1. Infants were randomly assigned to the following four groups: 1) a control group, including infants that were exposed to saline as a sham;2) an own mother's breast milk group (Own MM), including infants that were exposed to their mother's breast milk; 3) an other mother's breast milk group (Other MM), including infants that were exposed to other mother's breast milk; and 4) a formula milk group (Formula M), including infants that were exposed to formula milk. These four independent groups were homogeneous for sex, $\chi^{2}=0.8, p=0.85$; gestational age, $F(3,44)=0.63, p=0.59$; birth weight, $F(3,44)=0.76, p=0.52 ;$ primiparae / multiparae, $\chi^{2}=1.8, p=0.60$; vaginal / cesarean, $\chi^{2}=2.7, p=0.42$. The infant delivered by Caesarian section are not evenly distributed among groups $(0 \sim 15 \%)$, which might affect pain responses to odors. It is, however, unlikely because there is no difference among groups in birth weight, gestational age and Apgar score.

\section{Experiment 2}

For salivary cortisol measurements, we recruited 26 infants (15 boys, 11 girls) whose general characteristics, as well as those of their mothers, are given in Table 2. The selection criteria of these subjects were the same as those described above. Infants were randomly 
assigned to the following two groups: 1) a control group, including infants that were exposed to saline as a sham; and 2) an own mother's breast milk group (Own MM), including infants that were exposed to their mother's breast milk. These two independent groups were homogeneous for sex, $\chi^{2}=2.73, p=0.1$; gestational age, $t(24)=0.008, p=0.99$; birth weight, $t(24)=-0.17, p=0.86$; primiparae / multiparae, $\chi^{2}=0.004, p=0.95 . \quad$ All infants were born by vaginal delivery.

After the purpose of the experiment was explained to the parents of each infant, and they gave written informed consent. The protocol observed the tenets of the Helsinki Declaration and was approved by the Ethics Committee, Nagasaki University Graduate School of Biomedical Sciences.

\section{2. Stimuli}

Three milk stimuli were used: mother's breast milk, another mother's breast milk and

formula milk. Breakfast for the mothers was kept homogeneous in nutritional composition among mothers. Breast milk of each mother was sampled by manual extraction into plastic tubes $(15 \mathrm{ml}$; sample volume range $=2-5 \mathrm{ml})$. Under supervision by the attending physician, mother's milk was collected by the mother on day 5 postpartum. The milk samples were collected in the morning to minimize the influence of the mother's diet on variability in milk odor, because dietary odors pass into milk within 2 to $4 \mathrm{hr}$ of intake (Mennella and 
Beauchamp, 1991). Each sample was used in only one test. We used ready-to-use

formulas based on bovine milk given to bottle-fed newborns (Hagukumi ${ }^{\circledR}$, Morinaga Milk Industry CO., LTD.).

\section{3. Procedures}

\section{Experiment 1}

We observed infant behaviors before and after blood collection for congenital metabolic disease screening (Guthrie test) prescribed by the attending physician. The blood test is a routine medical procedure for all Japanese neonates, and was not prescribed for the purpose of the present study. The blood test was administered via a capillary puncture on the heel (heelstick) during the period 10:00-13:00 by the attending physician. Each infant was brought individually to a quiet room isolated from the sounds of other infants crying and placed on a bed warmed to $37^{\circ} \mathrm{C}$. The room temperature and light conditions were kept constant at around $25^{\circ} \mathrm{C}$ and around $80 \mathrm{~lx}$, respectively. The experiment was started 3 min before the heelstick, and continued until 9 min after the heelstick.

The attending physician held up the baby's heel and pricked the heel to collect the blood sample. After the heelstick, the attending physician placed a Band-Aid ${ }^{\mathrm{TM}}$ on each infant's limb to stop bleeding. The mean number of heelsticks was $1.6 \pm 0.1$ and the mean duration of the heelsticks was $202 \pm 20 \mathrm{sec}$ (see Table 1). No significant difference was 
found among the groups in the number or duration of heelsticks $(F(3,3.0)=0.82, p=0.49$

for number of heelsticks; $F(3,2.9)=0.98, p=0.42$ for duration of heelsticks $)$. Moreover, no correlation was found between the number of heelsticks and crying, grimacing and motor activity after the heelstick ( $r=0.02,-0.01$ and -0.08 , respectively). Sim ilarly, no correlation was found between the duration of heelsticks and crying, grimacing and motor activity after the heelstick ( $r=-0.14,-0.13$ and -0.30 , respectively). After the experiment, infants were brought back to their own cot in their room.

During the experiment, infants wore an Actigraph (micro-mini Actigraph, A.M.I.) on their left arm to record motor activity. The experimenter also recorded an overhead view of infants' faces and upper bodies by digital video camera (DCR-DVD201, Sony) during the experiment.

For odorant application, the experimenter prepared $2 \mathrm{ml}$ of each odor sample in a $40 \mathrm{ml}$ glass vial, but the attending physician was not informed what odor would be offered. The glass vials containing the odor samples were kept warmed at $37{ }^{\circ} \mathrm{C}$ in a water bath during the exposure. Using a mini pump (MP-2N, Sibata Scientific Technology, Ltd.), air directed through the sampled glass vial was applied at a flow rate of $2.0 \mathrm{~L} / \mathrm{min}$ at a distance of $10 \mathrm{~cm}$ from the infant's nose during the experiment. The odor exposure was started 3 min before the heelstick, and continued until 9 min after the heelstick.

\section{Experiment 2}


The procedures were the same as those used in experiment 1 and no group differences were observed (see Table 2). Saliva samples were collected from infants by the attending physician using a Sorbette ${ }^{\circledR}$ (Salimetrics LLC) for $60 \mathrm{sec}$ periods in each time point (Lewis et al., 1993). First saliva sampling was started 1 min before the beginning of the odor exposure. Second sampling was performed 20 min after the beginning of the heelstick. The attending physician who collected saliva was not informed what odor would be offered to the infant. After saliva collection, the Sorbette ${ }^{\circledR}$ was inserted into a 2-ml cryovial and centrifuged for 20 min at $3000 \mathrm{rpm}$ to extract fluid in the Sorbette ${ }^{\circledR}$. As a result, the mean extracted volume of saliva was $86.8 \pm 6.0 \mu \mathrm{l}$. Extrac ted saliva samples were frozen and kept at $-80{ }^{\circ} \mathrm{C}$ until assay. Saliva samples were measured using an EIA kit (expanded range high sensitivity salivary cortisol EIA kit, Salimetrics LLC, PA, US).

\section{4. Data Scoring}

Crying and grimacing were measured in 3-min bins by watching infant behavior on the video player. These data were coded by technicians. The coder was blind to the group assignment and type of stimulus. Motor activity was measured in 3-min bins using software (AW-2, A.M.I.).

\section{4. 1. Crying}


Scores coded crying second-to-second. Crying was defined as the presence of a crying sound lasting more than $1 \mathrm{sec}$. Data were converted into percentages for data analyses.

\section{4. 2. Grimacing}

Coding of the face was derived from Grunau and Craig (Grunau and Craig, 1987) and focused on three facial actions, namely, brow bulge, eye squeeze, and deepening of the nasolabial furrow. A grimace was the co-occurrence of these three facial actions, and was coded second-to-second as present or absent (Gray et al., 2000). Data were converted into percentages for data analysis.

\section{4. 3. Motor activity}

Motor activity was measured via arm movements by means of the actigraph. While increased motor activity is not a specific index of pain, it is a component of the pain response.

\section{5. Behavioral States}

Behavioral states were coded according to the classification of Prechtl (Prechtl, 1974).

The five states were grouped into three categories: asleep (State 1 and 2), awake (State 3 and

4), and fussy (State 5). The experimental groups were homogeneous for state, $\chi^{2}=4.76, p=$ 
0.57. Moreover, there was no effect of state across groups on crying, grimacing and motor activity during the baseline period. Twenty six infants were awake when the test was administered. Nine infants were excluded due to continuous sleeping or no crying (defined as no crying for at least $30 \mathrm{sec}$ after the heelstick).

\section{6. Statistical Analysis}

Data are presented as means \pm standard error (SE). To assess the differential effect of odor stimuli on the distress, two-way repeated measures ANOVAs were computed. Group (Control, Own MM, Other MM and Formula M) and time (0 to 3 min, 3 to 6 min, 6 to 9 min and 9 to $12 \mathrm{~min}$ ) were considered as potential determinants of the distress responses. When significant interactions were detected, subsequent analyses of simple main effects were conducted. Where overall effects were reported, these were followed by Tukey's HSD post hoc tests, comparing pairs of groups. For salivary cortisol measurement, we used paired t-test for before and after heelstick from the same infants. All analyses were done with SPSS 15.0 (SPSS Inc.).

\section{Results}

\section{Experiment 1}

\subsubsection{Crying data}


As shown in Fig. 1A, crying was increased in response to the heelsticks $[F(3,132)=$ 39.37, $p<0.0000]$. However, there was a significant interaction between group and time $[F$ $(9,132)=2.3, p<0.05]$. Subsequent analyses showed significant differences between the groups for 6 to $9 \min [F(3,176)=2.8]$ and 9 to $12 \min [F(3,176)=6.3]$. Post hoc analyses revealed significantly lower scores for the Own MM group compared with the Other MM $(p<0.05)$ group at 6 to 9 min and Control, Other MM and Formula M groups $(p<0.05)$ at 9 to $12 \mathrm{~min}$. No other pairwise comparisons yielded significance. There were significant differences in time course for Control $[F(3,132)=10.9]$, Own $\operatorname{MM}[F(3,132)=$ 11.1], Other $\operatorname{MM}[F(3,132)=18.5]$ and Formula $\mathrm{M}[F(3,132)=5.7]$ groups. Post hoc analyses revealed a greater increase in crying at 3 to 6 min than 0 to $3 \mathrm{~min}$ in all groups $(p<$ 0.05). In Control, Other MM and Formula M groups, crying remained at higher levels at 6 to $9 \min$ and 9 to $12 \min$ than at 0 to $3 \min (p<0.05)$. However, in the Own MM group, there were no significant increases in crying in these periods, and there was a significant decrease at 9 to 12 min compared with the 3 to 6 min time bin $(p<0.05)$ (Fig. 1A). No other pair wise comparisons yielded significance.

\subsubsection{Grimacing data}

Grimacing and crying were highly correlated measures in this study $(r=0.99)$.

Grimacing increased in response to the heelsticks $[F(3,132)=39.37, p<0.0000]$. However, 
there was a significant interaction between group and time $[F(9,132)=2.6, p<0.01]$.

Subsequent analyses showed significant differences between the groups for 0 to $3 \min [F$ (3, $176)=3.3], 6$ to $9 \min [F(3,176)=3.4]$ and 9 to $12 \min [F(3,176)=7.0]$ time bins. Post hoc analyses revealed significant decreases in grimacing in the Own MM group compared with the Control, Other MM and Formula M groups $(p<0.05)$ at 6 to 9 min and 9 to 12 min. No other pairwise comparisons yielded significance. There were significant differences in time course for Control $[F(3,132)=12.0]$, Own MM $[F(3,132)=12.0]$, Other MM $[F(3$, $132)=21.5]$ and Formula $M[F(3,132)=5.2]$ groups. Post hoc analyses revealed a greater increase in grimacing at 3 to $6 \mathrm{~min}$ than at 0 to $3 \mathrm{~min}$ in all groups $(p<0.05)$. In the Control and Other MM groups, grimacing remained at higher levels in the 6 to 9 min and 9 to 12 min time bins than in the 0 to 3 min time bin $(p<0.05)$. In the Formula $M$ group, there were marginally significant increases in grimacing in these periods $(p<0.10)$. However, in the Own MM group, there were no significant increases in these periods, and there was a significant decrease in the 9 to 12 min time bin compared with the 3 to $6 \min (p<0.05)$ (Fig 1B). No other pairwise comparisons yielded significance.

\subsubsection{Motor Activity}

Motor activity were also highly correlated with crying $(r=0.82)$ and grimacing $(r=0.84) . \quad$ Motor activity increased in response to the heelsticks $[F(3,132)=39.37, p<$ 
0.0000]. However, there was a significant interaction between group and time $[F(9,111)=$ $2.2, p<0.05]$. Subsequent analyses showed significant differences between the groups in the 3 to $6 \min [F(3,148)=3.3], 6$ to $9 \min [F(3,148)=3.6]$ and 9 to $12 \min [F(3,148)=$ 5.1] time bins. Post hoc analyses revealed significant decreases in motor activity in the Own MM group compared with the Control and Other MM groups $(p<0.05)$ at 6 to 9 min and the Control, Other MM and Formula M groups $(p<0.05)$ at 9 to 12 min. No other pairwise comparisons yielded significance. T here were significant differences in time course for Control $[F(3,111)=7.0]$ and Other $\operatorname{MM}[F(3,111)=10.0]$ groups. Post hoc analyses revealed a greater increase in motor activity in the 3 to 6 min time bin than in the 0 to 3 min time bin in the Control and Other MM groups $(p<0.05)$. In the Control and Other MM groups, motor activity remained at a high level in the 6 to 9 min and 9 to 12 min time bins compared with the 0 to $3 \min (p<0.05)$ (Fig 1C). No other pairwise comparisons yielded significance.

\section{Experiment 2}

\subsubsection{Salivary cortisol measurement}

The mean of salivary cortisol value for the Control group was $11.7 \mathrm{nmol} / \mathrm{L}(\mathrm{SE}=2.0)$ before heelstick and $25.6 \mathrm{nmol} / \mathrm{L}(\mathrm{SE}=4.2)$ after heelstick. The mean of salivary cortisol value for the Own MM group was $19.0 \mathrm{nmol} / \mathrm{L}(\mathrm{SE}=4.0)$ before heelstick and $20.7 \mathrm{nmol} / \mathrm{L}$ (SE $=2.7$ ) after heelstick. Paired $t$-test revealed a significant increase in salivary cortisol levels 
after heelsticks compared with those before heelsticks $(t(13)=-2.72, p<0.05)$ in the Control group (Fig 2A), but not in Own MM group $(t(11)=-0.38)($ Fig 2B). 


\section{Discussion}

In the present study, we examined the effects of various milk odors on the pain responses of infants in order to explore the possibility of analgesia by milk odors as an alternative medicine. W e showed that behavioral indices of the pain response (crying, grimacing and motor activity) were decreased by the presentation of odors from infants' own mother's milk, but not those from other mother's milk or formula milk. We also found that the increase in a biochemical index of pain response (salivary cortisol level) was inhibited by exposure of infants to their mother's milk odors. These findings suggest that a mother's milk odors relieve pain in her own newborn, but not in other newborns.

\section{1. Soothing effect of mother's milk odors}

In the present study, we measured crying, grimacing and motor activities during heelsticks to assess infants' pain responses. As a result, infants exposed to their mother's milk odor showed less crying, grimacing and motor activity after the heelstick than control infants. Furtherm ore, we assessed the effect of mother's milk on pain responses by measuring cortisol levels in infant saliva. Increases in salivary cortisol levels in infants after the heelstick were suppressed by their mothers' milk odors. From these behavioral and biochemical results, we can safely suggest that the mother's breast milk odors have soothing effects on infants. 
Mellier and colleagues presented newborn infants with maternal milk odors during a

blood draw on the dorsum of the hand, and found that infants cried less when exposed to their mother's milk odor compared with infants exposed to odorless saline (Mellier et al., 1997). Rattaz and colleagues (2005) also showed the soothing effects of maternal milk odors on the head movements of infants during the heelstick. These results are consistent with the results of the present study.

Compared with previous studies, there are four unique points in the present study. First, we performed the experiment on postnatal day 5, when the olfactory preference has changed from the infant's amniotic fluid to their maternal milk odor (Marlier et al., 1997), although previous studies performed experiments on postnatal day 3 (Mellier et al., 1997; Rattaz et al., 2005). Secondly, all infants recruited in the present study were breast fed, but subjects recruited in a previous study (Mellier et al., 1997) included bottle-fed infants in addition to breast-fed infants. Thirdly, we added pain indices that were different from those used in previous studies; we measured salivary cortisol levels as a biochemical index and motor activity estimated by actigraph as a behavioral index. Lastly, we exposed infants to odors by means of constant air flow warmed to $37^{\circ} \mathrm{C}$, although air flow and its temperature were not regulated in previous studies (Mellier et al., 1997; Rattaz et al., 2005).

Amniotic fluid odors and maternal odors other than the breast milk odors also seem to have soothing effects on infants (Sullivan and Toubas, 1998). Spontaneous crying was 
reduced by the presentation of amniotic fluid (Varendi et al., 1998) and the hospital gown worn by the mother (Sullivan and Toubas, 1998). However, the soothing effect was examined only immediately after birth. We should determine whether amniotic fluid odors and mother's body odors relive pain in infants at postnatal day 5 .

\section{2. No soothing effect of other mother's milk odor}

In the present study, mothers' milk odors reduced the pain responses in their own infants, but not in other infants, suggesting that the soothing effect of maternal milk odors is restricted to a mother's own infant. Why do maternal milk odors have a soothing effect on a mother's own infant, but not other infants?

One possible explanation for the restriction of the soothing effect may be major histocompatibility complex (MHC)-based odor differences. MHC genes, which are involved in the adaptive immune response, also produce odor information about individuality and influence social behaviors (Beauchamp and Yamazaki, 2003; Wedekind et al., 1995). Infants, therefore, may discriminate MHC-based odors included in the maternal milk and respond exclusively to their own mother's milk. It is also possible that the odor familiarity to infants provides analgesia. Thus, Rattaz and colleagues (2005) showed that smelling of vanilla reduced agitation during the heelstick and diminished distress after the procedure when vanilla odors were familiar to infants. Before any conclusions can be drawn, we should 
determine whether or not infants' pains are relieved by milk odors from unfamiliar mothers with MHC genes highly-matched to those of the infant.

In contrast to our present results, a mother's body odors decreased spontaneous crying not only in her own infant, but also in other infants at 2 days of age, indicating that the soothing effects of maternal body odor are not restricted to a mother's own infant (Sullivan and Toubas, 1998). This inconsistency between the present and previous studies could be explained in two different ways. First is the age of infants participating in the study: 5-day-old infants in the present study and around 2-day-old infants in the previous study. This difference may account for the inconsistency, because olfactory-based responses in newborns may involve both biologically and prenatal / postnatal experience-dependent factors (Lipsitt, 1990; Mennella and Beauchamp, 1991; Varendi et al., 1996). Perinatal olfactory continuity was observed at 2 days of age since 2-day-old breast-fed newborns did not distinguish between the odors of their own amniotic fluid and those of their mother's colostrum when presented simultaneously (Marlier et al., 1998). Second are differences in odor source. Two-week-old girls who had been exclusively bottle-fed since birth spent more time oriented toward the breast odor of an unfamiliar lactating mother than to the same woman's axillary odor (Makin and Porter, 1989). It is interesting to note that breast-fed infants begin to orient discriminatively to their own mother's axillary odor at 2 weeks of age after becoming familiar with these odors, whereas maternal body odors indiscriminatively 
soothe their own and other infants at 2 days of age when infants can become familiar to soothing odors. By contrast, odor preference and the soothing effects of maternal milk odor are already discriminative at around 5 days old, as shown in the present study and a previous study (Macfarlane, 1975).

\section{3. Formula milk odor}

In the present study, we found that formula milk did not relieve heelstick pain in infants. Mellier and colleagues also showed that maternal milk was more effective in pain relief than formula milk (Mellier et al., 1997). A recent study also investigated the effects of artificial milk odors on infants' behavioral and physiological reactions to routine heelstick procedures. Neither grimacing nor crying measures differed among infants exposed to the scent of linalyl acetate, artificial milk odor, or air in an odorless control condition. The authors pointed out that the artificial milk odor composed of $\gamma$-dodecalactone was not enough to soothe infants because of indiscriminative behavioral / physiological responses to the artificial milk odors (Kawakami et al., 1997).

Odor preference for formula milk was examined in a previous study (Marlier and Schaal, 2005). Newborns were attracted to maternal milk odors more than formula milk odors, and this preference was independent of postnatal feeding experience (Marlier and Schaal, 2005). However, formula milk odors were preferred when compared with odorless 
saline. This phenomenon might be explained by the fact that infants could become familiar with formula milk odors. If so, it is possible that the odor familiarity to formula milk is not effective for pain relief, although vanilla, an artificial odor, is effective for pain relief after infants have became familiar with the odor. We should examine the soothing effects of formula milk and vanilla on bottle-fed infants and compare their effects.

\section{Conclusions}

Using various behavioral and biochemical indices, we showed that infant pain responses were decreased by odors from an infant's mother's milk, but not by odors from other mothers' milk or formula milk. This suggests that the soothing effects of maternal milk odors depend on whether the odor is originated from an infant's own mother. The phenomenon that maternal milk odors are effective for pain relief only in a mother's own infant is very interesting, but its mechanism could not been clarified in the present study.

\section{Acknowledgments}

This research was supported financially by the Research Institute of Science and Technology for Society (RISTEX), Japan Science and Technology Agency (JST). We are grateful to the medical staff of the clinic and hospital for technical help. 


\section{References}

Beauchamp, G.K., Yamazaki, K., 2003. Chemical signalling in mice. Biochem Soc Trans 31, $147-151$.

Cernoch, J.M., Porter, R.H., 1985. Recognition of maternal axillary odors by infants. Child Dev 56, 1593-1598.

Craig, K.D., Whitfield, M.F., Grunau, R.V., Linton, J., Hadjistavropoulos, H.D., 1993. Pain in the preterm neonate: behavioural and physiological indices. Pain 52, 287-299.

Gray, L., Watt, L., Blass, E.M., 2000. Skin-to-skin contact is analgesic in healthy newborns. Pediatrics 105, e14.

Grunau, R.V., Craig, K.D., 1987. Pain expression in neonates: facial action and cry. Pain 28, $395-410$.

Johnston, C.C., Filion, F., Snider, L., Majnemer, A., Limperopoulos, C., Walker, C.D., Veilleux, A., Pelausa, E., Cake, H., Stone, S., Sherrard, A., Boyer, K., 2002. Routine sucrose analgesia during the first week of life in neonates younger than 31 weeks' postconceptional age. Pediatrics 110, 523-528.

Johnston, C.C., Stevens, B., Yang, F., Horton, L., 1996. Developmental changes in response to heelstick in preterm infants: a prospective cohort study. Dev Med Child Neurol 38, 438-445.

Johnston, C.C., Stevens, B.J., Yang, F., Horton, L., 1995. Differential response to pain by very premature neonates. Pain 61, 471-479. 
Kawakami, K., Kawakami, K., Okazaki, Y., Kurihara, H., Shimizu, Y., Yanaihara, T., 1997.

The Effect of odors on human newborn infants under stress. Infant Bheavior \& Development $20,531-535$.

Lewis, M., Ramsay, D.S., Kawakami, K., 1993. Differences between Japanese infants and Caucasian American infants in behavioral and cortisol response to inoculation. Child Dev 64, $1722-1731$.

Lipsitt, L.P., 1990. Learning processes in the human newborn. Sensitization, habituation, and classical conditioning. Ann N Y Acad Sci 608, 113-123; discussion 123-117.

Macfarlane, A., 1975. Olfaction in the development of social preferences in the human neonate. Ciba Found Symp, 103-117.

Makin, J.W., Porter, R.H., 1989. Attractiveness of lactating females' breast odors to neonates.

Child Dev 60, 803-810.

Marlier, L., Schaal, B., 2005. Human newborns prefer human milk: conspecific milk odor is attractive without postnatal exposure. Child Dev 76, 155-168.

Marlier, L., Schaal, B., Soussignan, R., 1997. Orientation responses to biological odours in the human newborn. Initial pattern and postnatal plasticity. C R Acad Sci III 320, 999-1005. Marlier, L., Schaal, B., Soussignan, R., 1998. Neonatal responsiveness to the odor of amniotic and lacteal fluids: a test of perinatal chemosensory continuity. Child Dev 69, 611-623. Mellier, D., Be` zard, S., J, C., 1997. E'tudes exploratoires des relations intersensorielles 
olfaction-douleur. Enfance 1, 47-64.

Mennella, J.A., Beauchamp, G.K., 1991. Maternal diet alters the sensory qualities of human milk and the nursling's behavior. Pediatrics $88,737-744$.

Prechtl, H.F., 1974. The behavioural states of the newborn infant (a review). Brain Res 76, $185-212$.

Rattaz, C., Goubet, N., Bullinger, A., 2005. The calming effect of a familiar odor on full-term newborns. J Dev Behav Pediatr 26, 86-92.

Russell, M.J., 1976. Human olfactory communication. Nature 260, 520-522.

Schaal, B., Montagner, H., Hertling, E., Bolzoni, D., Moyse, A., Quichon, R., 1980.

[Olfactory stimulation in the relationship between child and mother]. Reprod Nutr Dev 20, $843-858$

Sullivan, R.M., Toubas, P., 1998. Clinical usefulness of maternal odor in newborns: soothing and feeding preparatory responses. Biol Neonate 74, 402-408.

Varendi, H., Christensson, K., Porter, R.H., Winberg, J., 1998. Soothing effect of amniotic fluid smell in newborn infants. Early Hum Dev 51, 47-55.

Varendi, H., Porter, R.H., Winberg, J., 1994. Does the newborn baby find the nipple by smell? Lancet 344, 989-990.

Varendi, H., Porter, R.H., Winberg, J., 1996. Attractiveness of amniotic fluid odor: evidence of prenatal olfactory learning? Acta Paediatr 85, 1223-1227. 
Wedekind, C., Seebeck, T., Bettens, F., Paepke, A.J., 1995. MHC-dependent mate preferences in humans. Proc Biol Sci 260, 245-249.

Winberg, J., Porter, R.H., 1998. Olfaction and human neonatal behaviour: clinical implications. Acta Paediatr 87, 6-10. 


\section{Figure legends}

Figure 1. (A) The mean ( $\pm \mathrm{SE}$ ) duration of crying in response to heelsticks as a pain index. a: $p<0.05$ (Own MM vs. Control). b: $p<0.05$ (Own MM vs. Other MM). c: $p<0.05$ (Own MM vs. Formula M). $\quad *: p<0.05$ (vs. 0 to 3 min). $\quad *: p<0.05$ ( 3 to 6 min vs. 9 to 12 min in Own MM). (B) The mean $( \pm \mathrm{SE})$ duration of grimacing in response to the heelsticks as a pain index. a: $p<0.05$ (Own MM vs. Control). b: $p<0.05$ (Own MM vs. Other MM). c: $p<0.05$ (Own MM vs. Formula M). $\quad *: p<0.05$ (vs. 0 to $3 \mathrm{~min}$ ). $\quad *: p<0.05$ (3 to 6 min vs. 9 to 12 min in Own MM). \#: $p<0.10$ (vs. 0 to 3 min). (C) The mean ( \pm SE) motor activity in response to the heelsticks as a pain index. a: $p<0.05$ (Own MM vs Control), b: $p$ $<0.05$ (Own MM vs Other MM), c: $p<0.05$ (Own MM vs Formula M). *: $p<0.05$ (vs. 0 to 3 min). Open circles: Control. Closed circles: Own MM. Open triangle with short-dashed line: Other MM. Open square with long-dashed line: Formula M.

Figure 2. (A) Individual salivary cortisol levels (nmol / L) before and after heelstick in Control group. *: $p<0.05$ (before vs. after heelstick). (B) Individual salivary cortisol levels (nmol / L) before and after heelstick in Own MM group. Solid lines indicate infants who had a lower cortisol after heelstick as compared to before heelstick. Dotted lines indicate infants who did not have a reduction in cortisol after heelstick as compared to before heelstick. 
Table 1

Summary information for each group in experiment 1.

\begin{tabular}{|c|c|c|c|c|c|}
\hline & Control $(n=12)$ & Own MM $(n=12)$ & Other MM $(n=15)$ & Formula M $(n=9)$ & Total $(n=48)$ \\
\hline \multicolumn{6}{|l|}{ Infant } \\
\hline \multicolumn{6}{|l|}{ Sex } \\
\hline Male & 6 & 7 & 10 & 5 & 28 \\
\hline Female & 6 & 5 & 5 & 4 & 20 \\
\hline Gestational age (w) & $39.2 \pm 0.5$ & $39.9 \pm 0.3$ & $39.4 \pm 0.2$ & $39.8 \pm 0.3$ & $39.6 \pm 0.2$ \\
\hline Birth weight $(\mathrm{g})$ & $3092 \pm 126$ & $3184 \pm 85$ & $2985 \pm 88$ & $3106 \pm 91$ & $3084 \pm 50$ \\
\hline \multicolumn{6}{|l|}{ Heelsticks } \\
\hline Number & $1.3 \pm 0.3$ & $2.0 \pm 0.6$ & $1.5 \pm 0.2$ & $1.8 \pm 0.3$ & $1.6 \pm 0.1$ \\
\hline Duration (s) & $144 \pm 16$ & $219 \pm 43$ & $192 \pm 38$ & $243 \pm 39$ & $202 \pm 20$ \\
\hline \multicolumn{6}{|l|}{ Behavioral states } \\
\hline Asleep (state 1,2 ) & 6 & 5 & 9 & 2 & 22 \\
\hline Awake (state 3,4 ) & 3 & 4 & 3 & 4 & 14 \\
\hline Fussy (state 5) & 3 & 3 & 3 & 3 & 12 \\
\hline \multicolumn{6}{|l|}{ Mother } \\
\hline Primiparae/multiparae & $4 / 8$ & $6 / 6$ & $5 / 10$ & $5 / 4$ & $20 / 28$ \\
\hline Vaginal delivery/caesarian section & $11 / 1$ & $12 / 0$ & $13 / 2$ & $9 / 0$ & $45 / 3$ \\
\hline
\end{tabular}

Table 2

Summary information for each group in experiment 2 .

\begin{tabular}{llll}
\hline & $\begin{array}{l}\text { Control } \\
(n=14)\end{array}$ & $\begin{array}{l}\text { Own MM } \\
(n=12)\end{array}$ & $\begin{array}{l}\text { Total } \\
(n=26)\end{array}$ \\
\hline Infant & & & \\
Sex & 6 & 9 & 15 \\
$\quad$ Male & 6 & 3 & 11 \\
$\quad$ Female & $39.7 \pm 0.2$ & $39.7 \pm 0.2$ & $39.7 \pm 0.1$ \\
Gestational age (w) & $3163 \pm 48$ & $3188 \pm 70$ & $3174 \pm 41$ \\
Birth weight (g) & & & \\
Heelsticks & $2.3 \pm 0.2$ & $2.5 \pm 0.2$ & $2.4 \pm 0.1$ \\
$\quad$ Number & $296 \pm 19$ & $317 \pm 20$ & $306 \pm 13$ \\
$\quad$ Duration (s) & & & \\
Behavioral states & 7 & 8 & 15 \\
$\quad$ Asleep (state 1, 2) & 3 & 1 & 7 \\
$\quad$ Awake (state 3, 4) & 4 & 3 & $15 / 11$ \\
$\quad$ Fussy (state 5) & & & $26 / 0$ \\
$\quad$ Mother & $8 / 6$ & $7 / 5$ & \\
Primiparae/multiparae & $14 / 0$ & $12 / 0$ & \\
Vaginal delivery/caesarian section & & &
\end{tabular}

